Pacific

Journal of

Mathematics

THE KERNEL OF FOCK REPRESENTATIONS OF WICK ALGEBRAS WITH BRAIDED OPERATOR OF COEFFICIENTS

Palle E. T. Jørgensen, Danill P. Proskurin, and Yurĭ S.

SAMOILLENKO

Volume $198 \quad$ No. 1

March 2001 


\title{
THE KERNEL OF FOCK REPRESENTATIONS OF WICK ALGEBRAS WITH BRAIDED OPERATOR OF COEFFICIENTS
}

\author{
Palle E. T. Jørgensen, Danill P. Proskurin, and Yurĭ S. \\ SAMOĬLENKO
}

It is shown that the kernel of the Fock representation of a certain Wick algebra with braided operator of coefficients $T,\|T\| \leq 1$, coincides with the largest quadratic Wick ideal. Improved conditions on the operator $T$ for the Fock inner product to be strictly positive are given.

\section{Introduction.}

The problem of positivity of the Fock space inner product is central in the study of the Fock representation of Wick algebras (see [2], [3], [5], [6]). The paper [6] presents several conditions on the coefficients of the Wick algebra for the Fock inner product to be positive. If the operator of coefficients of the Wick algebra $T$ satisfies the braid condition and the norm restriction $\|T\| \leq 1$, then, as proved in [2], the Fock inner product is positive. Moreover if $-1<T<1$, it was shown in [2] that the Fock inner product is strictly positive. In this article we prove that, for braided $T$ with $\|T\| \leq 1$, the kernel of the Fock inner product coincides with the largest quadratic Wick ideal. In particular this implies that, for $-1<T \leq 1$, the Fock inner product is strictly positive definite, and the Fock representation is faithful.

This article is organized as follows. In Sec. 2 we present definitions of Wick algebras and the Fock representation and show that, in the braided case, the kernel of the Fock representation is generated by the kernel of the Fock inner product. In Sec. 3 we prove that if the operator $T$ is braided and $\|T\| \leq 1$, then the kernel of the Fock inner product coincides with the twosided ideal generated by $\operatorname{ker}(1+T)$. In Sec. 4 we combine results obtained in Sec. 2 and Sec. 3 to examine the $C^{*}$-representability of certain Wick algebras or their quotients. All results are illustrated by examples of different kinds of $q_{i j}$-CCR. 


\section{Preliminaries.}

For more detailed information about Wick algebras and the Fock representation we refer the reader to [6]. In this section we present only the basic definitions and properties.

1. The notion of a $*$-algebra allowing Wick ordering (Wick algebra) was presented in the paper [6] as a generalization of a wide class of $*$-algebras [7], including the twisted CCR and CAR algebras (see [10]), the $q$-CCR (see [4]) algebra, etc.

Definition 1. Let $\mathbb{J}=\mathbb{J}_{d}=\{1,2, \ldots, d\}, T_{i j}^{k l} \in C, i, j, k, l \in \mathbb{J}$, be such that $T_{i j}^{k l}=\overline{T_{j i}^{l k}}$. The Wick algebra with the set of coefficients $\left\{T_{i j}^{k l}\right\}$ is denoted $W(T)$, and is a $*$-algebra, defined by generators $a_{i}, a_{i}^{*}, i \in \mathbb{J}$, which satisfy the basic relations:

$$
a_{i}^{*} a_{j}=\delta_{i j} 1+\sum_{k, l=1}^{d} T_{i j}^{k l} a_{l} a_{k}^{*} .
$$

Definition 2. Monomials of the form $a_{i_{1}} a_{i_{2}} \cdots a_{i_{m}} a_{j_{1}}^{*} a_{j_{2}}^{*} \cdots a_{j_{k}}^{*}$ are called Wick ordered monomials.

It was proved in $[6]$ that the Wick ordered monomials form a basis for $W(T)$.

Let $\mathcal{H}=\left\langle e_{1}, \ldots, e_{d}\right\rangle$. Consider the full tensor algebra over $\mathcal{H}, \mathcal{H}^{*}$, denoted by $\mathcal{T}\left(\mathcal{H}, \mathcal{H}^{*}\right)$. Then

$$
W(T) \simeq \mathcal{T}\left(\mathcal{H}, \mathcal{H}^{*}\right) /\left\langle e_{i}^{*} \otimes e_{j}-\delta_{i j} 1-\sum T_{i j}^{k l} e_{l} \otimes e_{k}^{*}\right\rangle .
$$

To study the structure of Wick algebras, and the structure of the Fock representation, it is useful to introduce the following operators on $\mathcal{H}^{\otimes n}:=$ $\underbrace{\mathcal{H} \otimes \cdots \otimes \mathcal{H}}_{n}($ see $[\mathbf{6}]):$

$$
\begin{gathered}
T: \mathcal{H} \otimes \mathcal{H} \mapsto \mathcal{H} \otimes \mathcal{H}, \quad T e_{k} \otimes e_{l}=\sum_{i, j} T_{i k}^{l j} e_{i} \otimes e_{j}, T=T^{*}, \\
T_{i}: \mathcal{H}^{\otimes n} \mapsto \mathcal{H}^{\otimes n}, \quad T_{i}=\underbrace{1 \otimes \cdots \otimes 1}_{i-1} \otimes T \otimes \underbrace{1 \otimes \cdots \otimes 1}_{n-i-1}, \\
R_{n}: \mathcal{H}^{\otimes n} \mapsto \mathcal{H}^{\otimes n}, \quad R_{n}=1+T_{1}+T_{1} T_{2}+\cdots+T_{1} T_{2} \cdots T_{n-1}, \\
P_{n}: \mathcal{H}^{\otimes n} \mapsto \mathcal{H}^{\otimes n}, \quad P_{2}=R_{2}, P_{n+1}=\left(1 \otimes P_{n}\right) R_{n+1} .
\end{gathered}
$$

In this article we suppose that the operator $T$ is contractive, i.e., $\|T\| \leq 1$, and satisfies the braid condition, i.e., on $\mathcal{H}^{\otimes 3}$ the equality $T_{1} T_{2} T_{1}=T_{2} T_{1} T_{2}$ holds. It follows from the definition of $T_{i}$ that then $T_{i} T_{j}=T_{j} T_{i}$ if $|i-j| \geq 2$, and for the braided $T$ one has $T_{i} T_{i+1} T_{i}=T_{i+1} T_{i} T_{i+1}$. 
Remark 1. These conditions hold for such well-known algebras as $q_{i j}$-CCR, $\mu$-CCR, $\mu$-CAR (see $[6]$ ).

The Fock representation of a Wick $*$-algebra is determined by a vector $\Omega$ such that $a_{i}^{*} \Omega=0$ for all $i=1, \ldots, d$ (see $[6]$ ).

Definition 3 (The Fock representation). The representation $\lambda_{0}$, acting on the space $\mathcal{T}(\mathcal{H})$ by formulas

$$
\begin{aligned}
\lambda_{0}\left(a_{i}\right) e_{i_{1}} \otimes \cdots \otimes e_{i_{n}} & =e_{i} \otimes e_{i_{1}} \otimes \cdots \otimes e_{i_{n}}, \quad n \in \mathbb{N} \cup\{0\}, \\
\lambda_{0}\left(a_{i}^{*}\right) 1 & =0,
\end{aligned}
$$

where the action of $\lambda_{0}\left(a_{i}^{*}\right)$ on the monomials of degree $n \geq 1$ is determined inductively using the basic relations, is called the Fock representation.

Note that the Fock representation is not a $*$-representation with respect to the standard inner product on $\mathcal{T}(\mathcal{H})$. However, it was proved in $[\mathbf{6}]$ that there exists a unique Hermitian sesquilinear form $\langle\cdot, \cdot\rangle_{0}$ on $\mathcal{T}(\mathcal{H})$ such that $\lambda_{0}$ is a $*$-representation on $\left(\mathcal{T}(\mathcal{H}),\langle\cdot, \cdot\rangle_{0}\right)$. This form is called the Fock inner product on $\mathcal{T}(\mathcal{H})$.

The subspaces $\mathcal{H}^{\otimes n}, \mathcal{H}^{\otimes m}, n \neq m$, are orthogonal with respect to $\langle\cdot, \cdot\rangle_{0}$, and on $\mathcal{H}^{\otimes n}$ we have the following formula (see [6]):

$$
\langle X, Y\rangle_{0}=\left\langle X, P_{n} Y\right\rangle, n \geq 2 \text {. }
$$

So, the positivity of the Fock inner product is equivalent to the positivity of operators $P_{n}, n \geq 2$, and $\mathcal{J}=\bigoplus_{n \geq 2}$ ker $P_{n}$ determines the kernel of the Fock inner product. It was noted in $[\mathbf{6}]$ that the Fock representation is the GNS representation associated with the linear functional $f$ on a Wick algebra such that $f(1)=1$ and, for any Wick ordered monomial, $f\left(a_{i_{1}} \cdots a_{i_{n}} a_{j_{1}}^{*} \cdots a_{j_{m}}^{*}\right)=$ 0 . Then for any $X, Y \in \mathcal{T}(\mathcal{H})$ we have (see $[6]$ ):

$$
\langle X, Y\rangle_{0}=f\left(X^{*} Y\right) \text {. }
$$

2. In the following proposition we describe the kernel of the Fock representation of a Wick algebra with braided operator $T$ in terms of the Fock inner product.

Proposition 1. Let $W(T)$ be the Wick algebra with braided operator $T$, and let the Fock representation $\lambda_{0}$ be positive (i.e., the Fock inner product is positive definite $)$. Then $\operatorname{ker} \lambda_{0}=\mathcal{J} \otimes \mathcal{T}\left(\mathcal{H}^{*}\right)+\mathcal{T}(\mathcal{H}) \otimes \mathcal{J}^{*}$.

Proof. First, we show that $X \in \operatorname{ker} P_{m}$ implies $X \in \operatorname{ker} \lambda_{0}$. Indeed, let $Y \in \mathcal{H}^{\otimes n}$; then

$$
\lambda_{0}(X) Y=X \otimes Y .
$$

Note that for braided $T$ we have the following decomposition (see [2] and Sec. 3 for more details):

$$
P_{n+m}=P\left(D_{m}\right)\left(P_{m} \otimes \mathbf{1}_{n}\right),
$$


where

$$
\begin{aligned}
& P\left(D_{m}\right)=\widetilde{R}_{n+m} \widetilde{R}_{n+m-1} \cdots \widetilde{R}_{m+1}, \\
& \widetilde{R}_{k}=1+T_{k-1}+T_{k-2} T_{k-1}+\cdots+T_{1} T_{2} \cdots T_{k-1}, \quad k \geq 2 .
\end{aligned}
$$

Then

$$
P_{n+m}\left(\lambda_{0}(X) Y\right)=P_{n+m}(X \otimes Y)=P\left(D_{m}\right)\left(P_{m} X \otimes Y\right)=0,
$$

and $\lambda_{0}(X)=0$ on $\left(\mathcal{T}(\mathcal{H}),\langle\cdot, \cdot\rangle_{0}\right)$. Therefore $\mathcal{J} \subset$ ker $\lambda_{0}$, and since ker $\lambda_{0}$ is a $*$-ideal,

$$
\mathcal{J} \otimes \mathcal{T}\left(\mathcal{H}^{*}\right)+\mathcal{T}(\mathcal{H}) \otimes \mathcal{J}^{*} \subset \operatorname{ker} \lambda_{0} .
$$

To prove the converse inclusion, we need a formula determining the action of $\lambda_{0}\left(X^{*}\right)$ on $\mathcal{T}(\mathcal{H})$ for any $X \in \mathcal{H}^{\otimes k}, k \in \mathbb{N}$. For $k=1, X=e_{i}, i=1, \ldots, d$, it was proved in $[6]$ that:

$$
\lambda_{0}\left(e_{i}^{*}\right) Y=\mu\left(e_{i}^{*}\right) R_{n} Y, \quad \forall Y \in \mathcal{H}^{\otimes n},
$$

where $\mu\left(e_{i}^{*}\right): \mathcal{T}(\mathcal{H}) \mapsto \mathcal{T}(\mathcal{H})$ is the annihilation operator:

$$
\mu\left(e_{i}^{*}\right) e_{i_{1}} \otimes e_{i_{2}} \otimes \cdots \otimes e_{i_{n}}=\delta_{i i_{1}} e_{i_{2}} \otimes \cdots \otimes e_{i_{n}} .
$$

Then, using the definition of $P_{n}$, it is easy to see that, for $X \in \mathcal{H}^{\otimes n}$ and $Y \in \mathcal{H}^{\otimes n}$,

$$
\lambda_{0}\left(X^{*}\right) Y=\left\langle X, P_{n} Y\right\rangle=\langle X, Y\rangle_{0} .
$$

Let now

$$
Z=\sum_{i=1}^{n} Y_{i} X_{i}^{*}+\sum_{j=n+1}^{l} Y_{j} X_{j}^{*} \in \operatorname{ker} \lambda_{0},
$$

where $Y_{i} \in \mathcal{T}(\mathcal{H}), i=1, \ldots, l$,

$$
X_{i} \in \mathcal{H}^{\otimes m}, i=1, \ldots, n, \quad X_{j} \in \mathcal{H}^{\otimes n_{j}}, n_{j}>m, \quad j=n+1, \ldots, l .
$$

Now (1) implies that we can suppose that the elements $X_{i}$ are linearly independent modulo J. Denote by $\left\{\widehat{X}_{i}, i=1, \ldots, n\right\} \subset \mathcal{H}^{\otimes m}$ a family dual to the $\left\{X_{i}, i=1, \ldots, n\right\}$ with respect to $\langle\cdot, \cdot\rangle_{0}$, i.e., such that

$$
\left\langle X_{i}, P_{m} \widehat{X}_{j}\right\rangle=\left\langle X_{i}, \widehat{X}_{j}\right\rangle_{0}=\delta_{i j}, \quad i, j=1, \ldots, n .
$$

Since, for any $j=n+1, \ldots, l$ and $i=1, \ldots, n$,

$$
\lambda_{0}\left(X_{j}^{*}\right) \widehat{X}_{i}=0,
$$

we have, in $\left(\mathcal{T}(\mathcal{H}),\langle\cdot, \cdot\rangle_{0}\right)$,

$$
0=\lambda_{0}(Z) \widehat{X}_{i}=Y_{i}, \quad i=1, \ldots, n,
$$

which implies $Y_{i} \in \mathcal{J}, i=1, \ldots, n$. The proof can be completed by evident induction. 
Remark 2. In particular, we have shown, for braided $T$, and for any $X \in$ $\mathcal{H}^{\otimes n}$ and $Y \in \operatorname{ker} P_{m}$, that

$$
X \otimes Y \in \operatorname{ker} P_{n+m} .
$$

By similar arguments, $Y \otimes X \in \operatorname{ker} P_{n+m}$, i.e., $\mathcal{J}=\left\langle\bigotimes_{n \geq 2} \operatorname{ker} P_{n}\right\rangle$ is a two-sided ideal in $\mathcal{T}(\mathcal{H})$.

The two-sided ideal $\mathcal{J} \subset \mathcal{T}(\mathcal{H})$ is called a Wick ideal (see [6]) if it satisfies the following condition:

$$
\mathcal{T}\left(\mathcal{H}^{*}\right) \otimes \mathcal{J} \subset \mathcal{J} \otimes \mathcal{T}\left(\mathcal{H}^{*}\right) .
$$

If $\mathcal{J}$ is generated by some subspace of $\mathcal{H}^{\otimes n}$, then $\mathcal{J}$ is called a homogeneous Wick ideal of degree $n$.

We show that for Wick algebras with braided operator of coefficients, J is a Wick ideal .

Proposition 2. Let $T$ satisfy the braid condition, and $\mathcal{J}=\left\langle\bigoplus_{n \geq 2} \operatorname{ker} P_{n}\right\rangle$; then

$$
\mathcal{H}^{*} \otimes \mathcal{J} \subset \mathcal{J}+\mathcal{J} \otimes \mathcal{H}^{*}
$$

Proof. Note that Conditions (2) and (3) are equivalent (see [6]). To prove the proposition, it is sufficient to show that, if $X \in \operatorname{ker} P_{n}$ for some $n \geq 2$, then for any $i=1, \ldots, d$,

$$
e_{i}^{*} \otimes X \in \operatorname{ker} P_{n-1}+\operatorname{ker} P_{n} \otimes \mathcal{H}^{*} .
$$

Indeed, for any $X \in \mathcal{H}^{\otimes n}$, we have the following formula (see [9]):

$$
e_{i}^{*} \otimes X=\mu\left(e_{i}^{*}\right) R_{n} X+\mu\left(e_{i}^{*}\right) \sum_{k=1}^{d} T_{1} T_{2} \cdots T_{n}\left(X \otimes e_{k}\right) \otimes e_{k}^{*} .
$$

Then for $X \in \operatorname{ker} P_{n}$, we have

$$
P_{n-1} \mu\left(e_{i}^{*}\right) R_{n} X=\mu\left(e_{i}^{*}\right)\left(1 \otimes P_{n-1}\right) R_{n} X=\mu\left(e_{i}^{*}\right) P_{n} X=0 .
$$

Note that, for braided $T$, for any $k=2, \ldots, n$,

$$
T_{k}\left(T_{1} T_{2} \cdots T_{n}\right)=\left(T_{1} T_{2} \cdots T_{n}\right) T_{k-1},
$$

which implies that

$$
\left(1 \otimes P_{n}\right)\left(T_{1} T_{2} \cdots T_{n}\right)=\left(T_{1} T_{2} \cdots T_{n}\right)\left(P_{n} \otimes 1\right) .
$$

Then for any $k=1, \ldots, d$,

$$
\begin{aligned}
P_{n} \mu\left(e_{i}^{*}\right) T_{1} T_{2} \cdots T_{n}\left(X \otimes e_{k}\right) & =\mu\left(e_{i}^{*}\right)\left(1 \otimes P_{n}\right) T_{1} T_{2} \cdots T_{n}\left(X \otimes e_{k}\right) \\
& =\mu\left(e_{i}^{*}\right) T_{1} T_{2} \cdots T_{n}\left(P_{n} \otimes 1\right)\left(X \otimes e_{k}\right)=0 .
\end{aligned}
$$


For Wick algebras with braided $T$, the largest homogeneous ideal of degree $n$ is generated by ker $R_{n}$ (see [6] and [9]), i.e., the condition ker $R_{n} \neq\{0\}$ is necessary and sufficient for the existence of homogeneous Wick ideals. In the following proposition we show that the same is true for arbitrary Wick ideals.

Theorem 1. If $\mathcal{J} \subset \mathcal{T}(\mathcal{H})$ is a non-trivial Wick ideal, then there exists $n \geq 2$ such that $\operatorname{ker} R_{n} \neq\{0\}$.

Proof. For any $X \in \mathcal{T}(\mathcal{H})$, by $\operatorname{deg} X$ we denote the highest degree of its homogeneous components. Let $Y \in \mathcal{J}$ be of minimal degree.

$$
Y=Y_{1}+Y_{2}+\cdots+Y_{k}, \quad Y_{i} \in \mathcal{H}^{\otimes n_{i}}, i=1, \ldots, k, n_{i} \in \mathbb{N} \cup\{0\} .
$$

Suppose that $\operatorname{deg} Y \geq 2$ : Then for any $i=1, \ldots, d$, we have

$$
e_{i}^{*} \otimes Y=\sum_{j=1}^{k} \mu\left(e_{i}^{*}\right) R_{n_{j}} Y_{j}+\mu\left(e_{i}^{*}\right) \sum_{j=1}^{k} \sum_{l=1}^{d} \widetilde{T}_{n_{j}}\left(Y_{j} \otimes e_{l}\right) \otimes e_{l}^{*},
$$

where we put $R_{0}=1, R_{1}=1$, and

$$
\widetilde{T}_{k}= \begin{cases}T_{1} T_{2} \cdots T_{k}, & k \geq 2 \\ T, & k=1 \\ 1, & k=0 .\end{cases}
$$

Then Condition (3) implies that for any $i=1, \ldots, d$,

$$
\sum_{j=1}^{k} \mu\left(e_{i}^{*}\right) R_{n_{j}} Y_{j} \in \mathcal{J} .
$$

Since the degrees of these elements are less than the degree of $Y$, we conclude that

$$
\sum_{j=1}^{k} \mu\left(e_{i}^{*}\right) R_{n_{j}} Y_{j}=0, \quad i=1, \ldots, d,
$$

and the independence of the Wick ordered monomials then implies

$$
\mu\left(e_{i}^{*}\right) R_{n_{j}} Y_{j}=0, \quad i=1, \ldots, d .
$$

Let $Y_{k}$ be the highest homogeneous component of $Y$; then, by our assumption, $\operatorname{deg} Y_{k} \geq 2$, and $\sum_{i=1}^{d} e_{i} \mu\left(e_{i}^{*}\right) R_{n_{k}} Y_{k}=R_{n_{k}} Y_{k}=0$, i.e., $Y_{k} \in \operatorname{ker} R_{n_{k}}$.

To complete the proof, note that if $X=\beta+\sum_{i=1}^{d} \alpha_{i} e_{i} \in \mathcal{J}$, then for any $j$, we have

$$
e_{j}^{*} \otimes X=\alpha_{j}+\beta e_{j}^{*}+\sum_{i=1}^{d} \alpha_{i} \sum_{k, l=1}^{d} T_{j i}^{k l} e_{l} \otimes e_{k}^{*},
$$

and (3) implies $\alpha_{j}=0, j=1, \ldots, d, \beta=0$. 


\section{The structure of ker $P_{n}$.}

In this section, we show that for Wick algebras with braided $T$ satisfying the condition $-1<T \leq 1$, the Fock representation is faithful, and for $-1 \leq T \leq 1$, the kernel of the Fock representation is generated by the largest quadratic Wick ideal (the largest quadratic Wick ideal is the largest homogeneous Wick ideal of degree 2).

To do this we need some properties of quasimultiplicative maps on the Coxeter group $S_{n}$ (for more detailed information we refer the reader to [2]).

1. Consider $S_{n+1}$ as a Coxeter group, i.e., a group defined as follows: $S_{n+1}=$ $\left\langle\sigma_{i}: \sigma_{i}^{2}=e, \sigma_{i} \sigma_{j}=\sigma_{j} \sigma_{i},|i-j| \geq 2, \sigma_{i} \sigma_{i+1} \sigma_{i}=\sigma_{i+1} \sigma_{i} \sigma_{i+1}, i=1, \ldots, n\right\rangle$. In order to study the invertibility of $P_{n}$ for any family of operators $\left\{T_{i}, i=\right.$ $1 \ldots, n, T \in B(\mathcal{K})\}$, satisfying the conditions

$$
T_{i} T_{i+1} T_{i}=T_{i+1} T_{i} T_{i+1}, T_{i}^{*}=T_{i},-1 \leq T_{i} \leq 1,
$$

where $\mathcal{K}$ is a separable Hilbert space, we may define (as in $[2]$ ) the function

$$
\phi: S_{n+1} \mapsto B(\mathcal{K})
$$

by the formulas

$$
\begin{gathered}
\phi(e)=1, \quad \phi\left(\sigma_{i}\right)=T_{i}, \\
\phi(\pi)=T_{i_{1}} \cdots T_{i_{k}},
\end{gathered}
$$

where $\pi=\sigma_{i_{1}} \cdots \sigma_{i_{k}}$ is a reduced decomposition. It was shown in [2] that

$$
P_{n+1}=P\left(S_{n+1}\right)=\sum_{\sigma \in S_{n+1}} \phi(\sigma) .
$$

Denote by $S$ the set of generators of $S_{n+1}$ as a Coxeter group. Consider, for any $J \subset S$, the set

$$
D_{J}=\left\{\sigma \in S_{n+1}|| \sigma s|=| \sigma \mid+1, \forall s \in J\right\} .
$$

Let $W_{J}$ be a Coxeter group, generated by $J$. Then $S_{n+1}=D_{J} W_{J}$ (see [1]), and $P_{n+1}=P\left(D_{J}\right) P\left(W_{J}\right)$ (see [2]). Using the equalities $P_{n+1}^{*}=P_{n+1}$, $P\left(W_{J}\right)^{*}=P\left(W_{J}\right)$, we obtain $P_{n+1}=P\left(W_{J}\right) P\left(D_{J}\right)^{*}$, where for all $M \subset$ $S_{n+1}$

$$
P(M)=\sum_{\sigma \in M} \phi(\sigma) .
$$

In what follows we use a quasimultiplicative analogue of the Euler-Solomon formula (see [2, Lemma 2.6]):

$$
\sum_{\substack{J \subset S \\ J \neq S, J \neq \emptyset}}(-1)^{|J|} P\left(D_{J}\right)=-(-1)^{|S|} 1+\phi\left(\sigma_{0}^{(n+1)}\right)-P\left(S_{n+1}\right),
$$


where $\sigma_{0}^{(n+1)}$ is the unique element of $S_{n+1}$ with maximal possible length of the reduced decomposition.

Remark 3. 1. The element $\sigma_{0}^{(n+1)}$ of the group $S_{n+1}$ has the form

$$
\sigma_{0}^{(n+1)}=\left(\sigma_{1} \cdots \sigma_{n}\right)\left(\sigma_{1} \cdots \sigma_{n-1}\right) \cdots\left(\sigma_{1} \sigma_{2}\right) \sigma_{1} .
$$

Set $U_{n}=\phi\left(\sigma_{0}^{(n+1)}\right)$ : Then

$$
U_{n}=\left(T_{1} T_{2} \cdots T_{n}\right)\left(T_{1} T_{2} \cdots T_{n-1}\right) \cdots\left(T_{1} T_{2}\right) T_{1} .
$$

2. It is easy to see that the operator $U_{n}$ is selfadjoint, and, taking adjoints, we can rewrite (6) in the following form:

$$
\sum_{J \subset S, J \neq \emptyset}(-1)^{|J|} P\left(D_{J}\right)^{*}=(-1)^{n+1} 1+U_{n}-P_{n+1} .
$$

3. Note also that, for all $J \subset S$, the group $W_{J}$ is isomorphic to $S_{k}$ for some $k<n$, or to the direct product of some such groups.

2. In what follows we shall use the following properties of the operator $U_{n}$.

Proposition 3. ker $P_{n+1}$ is invariant with respect to the action of $U_{n}$.

Proof. First we show that for all $J \subset S$,

$$
P\left(D_{J}^{*}\right): \operatorname{ker} P_{n+1} \mapsto \operatorname{ker} P_{n+1} .
$$

It can be easily obtained from the equality

$$
P_{n+1} P\left(D_{J}\right)^{*}=P\left(D_{J}\right) P\left(W_{J}\right) P\left(D_{J}\right)^{*}=P\left(D_{J}\right) P_{n+1} .
$$

Then by (7), we have

$$
U_{n}-(-1)^{n} 1: \operatorname{ker} P_{n+1} \mapsto \operatorname{ker} P_{n+1} .
$$

Proposition 4. Let operators $\left\{T_{i}, i=1, \ldots, n\right\}$ satisfy the braid condition $T_{i} T_{i+1} T_{i}=T_{i+1} T_{i} T_{i+1}, i=1, \ldots, n-1$, and $T_{i} T_{j}=T_{j} T_{i},|i-j| \geq 2$. Then

$$
T_{k} U_{n}=U_{n} T_{n+1-k}, \quad \forall k=1, \ldots n .
$$

Proof. 1. For $n=1$ the equality is evident.

2. Suppose that (8) holds for any $n \leq m$. Note that

$$
U_{m+1}=T_{1} T_{2} \cdots T_{m+1} U_{m} .
$$


Then, for $1<k \leq m+1$, we have

$$
\begin{aligned}
T_{k} U_{m+1} & =T_{k}\left(T_{1} T_{2} \cdots T_{m+1}\right) U_{m} \\
& =T_{1} T_{2} \cdots T_{k-2} T_{k} T_{k-1} T_{k} T_{k+1} \cdots T_{m+1} U_{m} \\
& =T_{1} T_{2} \cdots T_{k-2} T_{k-1} T_{k} T_{k-1} T_{k+1} \cdots T_{m+1} U_{m} \\
& =\left(T_{1} T_{2} \cdots T_{m+1}\right) T_{k-1} U_{m} \\
& =T_{1} T_{2} \cdots T_{m+1} U_{m} T_{m+1-(k-1)} \\
& =U_{m+1} T_{m+2-k} .
\end{aligned}
$$

In particular, for $k=m+1$ we have $T_{m+1} U_{m+1}=U_{m+1} T_{1}$. Then taking adjoints, we obtain the required equality for $k=1$.

3. Now we can formulate the main result of this paper.

Theorem 2. Let $W(T)$ be a Wick algebra with braided operator $T$ satisfying the norm bound $\|T\| \leq 1$. Then for any $n \geq 2$, we have

$$
\operatorname{ker} P_{n+1}=\sum_{k+l=n-1} \mathcal{H}^{\otimes k} \otimes \operatorname{ker}(1+T) \otimes \mathcal{H}^{\otimes l}=\sum_{k=1}^{n} \operatorname{ker}\left(1+T_{k}\right) .
$$

Proof. In fact, we shall prove the following: Let $T_{1}, T_{2}, \ldots, T_{n} \in B(\mathcal{K})$, where $\mathcal{K}$ is a finite-dimensional Hilbert space, be selfadjoint contractions satisfying the relations

$$
T_{i} T_{i+1} T_{i}=T_{i+1} T_{i} T_{i+1}, 1 \leq i \leq n-1, \quad T_{i} T_{j}=T_{j} T_{i},|i-j| \geq 2 .
$$

Then

$$
\operatorname{ker} P_{n+1}=\sum_{k=1}^{n} \operatorname{ker}\left(1+T_{k}\right)
$$

(It follows trivially from the decomposition $P_{n+1}=P\left(D_{\{k\}}\right)\left(1+T_{k}\right)$ that $\sum_{k=1}^{n} \operatorname{ker}\left(1+T_{k}\right) \subset \operatorname{ker} P_{n+1}$. $)$

We proceed using induction.

The case $n=2$.

In this case $P_{2}=1+T$.

The case $n \mapsto n+1$.

It follows from $P_{n+1}=P\left(W_{J}\right) P\left(D_{J}\right)^{*}$ that

$$
P\left(D_{J}\right)^{*}: \operatorname{ker} P_{n+1} \mapsto \operatorname{ker} P\left(W_{J}\right),
$$

i.e., $\operatorname{ran}\left(\left.P\left(D_{J}\right)^{*}\right|_{\operatorname{ker} P_{n+1}}\right) \subset \operatorname{ker} P\left(W_{J}\right)$. Moreover, it is obvious that for any $J \subset S$, ker $P\left(W_{J}\right) \subset \operatorname{ker} P_{n+1}$. Therefore, by (7), we have the following inclusion:

$$
\left.\operatorname{ran}\left(U_{n}-(-1)^{n} 1\right)\right|_{\operatorname{ker} P_{n+1}} \subset \sum_{J \subset S, J \neq \emptyset} \operatorname{ker} P\left(W_{J}\right)
$$


Since, for $J \subset S$, the group $W_{J}=W_{J_{1}} \times \cdots \times W_{J_{k}}$, where $W_{J_{l}} \simeq S_{n_{l}}$ with $n_{l}<n+1$, we have a decomposition into the product of pairwise commuting selfadjoint operators

$$
P\left(W_{J}\right)=P\left(W_{J_{1}}\right) \cdots P\left(W_{J_{k}}\right) .
$$

Therefore

$$
\operatorname{ker} P\left(W_{J}\right)=\sum_{l=1}^{k} \operatorname{ker} P\left(W_{J_{l}}\right) \subset \sum_{i=1}^{n} \operatorname{ker}\left(1+T_{i}\right) \text {, }
$$

where the last inclusion is obtained from the assumption of induction. So,

$$
\left.\operatorname{ran}\left(U_{n}-(-1)^{n} 1\right)\right|_{\operatorname{ker} P_{n+1}} \subset \sum_{i=1}^{n} \operatorname{ker}\left(1+T_{i}\right) .
$$

Consider the operator $1-U_{n}^{2}$. Since $U_{n}=U_{n}^{*}$ : $\operatorname{ker} P_{n+1} \mapsto \operatorname{ker} P_{n+1}$, then ker $P_{n+1}=\left.\operatorname{ran}\left(1-U_{n}^{2}\right)\right|_{\operatorname{ker} P_{n+1}}+\left.\operatorname{ker}\left(1-U_{n}^{2}\right)\right|_{\operatorname{ker} P_{n+1}}$.

Moreover, since $\operatorname{ran}\left(1-U_{n}^{2}\right) \subset \operatorname{ran}\left(U_{n}-(-1)^{n} 1\right)$, using (10), we have the inclusion

$$
\operatorname{ker} P_{n+1} \subset \sum_{k=1}^{n} \operatorname{ker}\left(1+T_{i}\right)+\left.\operatorname{ker}\left(1-U_{n}^{2}\right)\right|_{\operatorname{ker} P_{n+1}} \text {. }
$$

To finish the proof it remains only to show that

$$
\operatorname{ker}\left(1-U_{n}^{2}\right) \cap \operatorname{ker} P_{n+1} \subset \sum_{i=1}^{n} \operatorname{ker}\left(1+T_{i}\right) .
$$

To this end, we may present $1-U_{n}^{2}$ in the form

$$
\begin{aligned}
1-U_{n}^{2}= & 1-T_{1} T_{2} \cdots T_{n} U_{n-1}^{2} T_{n} \cdots T_{2} T_{1} \\
= & \left(1-T_{1}^{2}\right)+T_{1}\left(1-T_{2}^{2}\right) T_{1} \\
& +\cdots \\
& +T_{1} T_{2} \cdots T_{n-1}\left(1-T_{n}^{2}\right) T_{n-1} \cdots T_{2} T_{1} \\
& +T_{1} T_{2} \cdots T_{n}\left(1-U_{n-1}^{2}\right) T_{n} \cdots T_{2} T_{1} .
\end{aligned}
$$

Since $\|T\| \leq 1$ implies that $\left\|T_{i}\right\| \leq 1, i=1, \ldots n$, and $\left\|U_{k}\right\| \leq 1, k \geq 2$, then we have a sum of non-negative operators, and $v \in \operatorname{ker}\left(1-U_{n}^{2}\right)$ implies that

$$
\begin{aligned}
& T_{1}^{2} v=v, \\
& T_{2}^{2} T_{1} v=T_{1} v, \\
& \vdots \\
& T_{n}^{2} T_{n-1} \cdots T_{2} T_{1} v=T_{n-1} \cdots T_{2} T_{1} v .
\end{aligned}
$$

However, $T_{k} U_{n}=U_{n} T_{n+1-k}$ implies that $T_{k} U_{n}^{2}=U_{n}^{2} T_{k}$, and, consequently,

$$
T_{k}: \operatorname{ker}\left(1-U_{n}^{2}\right) \mapsto \operatorname{ker}\left(1-U_{n}^{2}\right), \quad k=1, \ldots, n .
$$


Moreover, since the restriction of $T_{1}$ to $\operatorname{ker}\left(1-U_{n}^{2}\right)$ is an involution,

$$
\left.\operatorname{ran}\left(T_{1}\right)\right|_{\operatorname{ker}\left(1-U_{n}^{2}\right)}=\operatorname{ker}\left(1-U_{n}^{2}\right),
$$

and, for any $v \in \operatorname{ker}\left(1-U_{n}^{2}\right)$, we have $T_{2}^{2} v=v$. By the same arguments, we obtain that

$$
\forall v \in \operatorname{ker}\left(1-U_{n}^{2}\right), \quad T_{i}^{2} v=v, i=1, \ldots, d .
$$

Let now $v \in \operatorname{ker}\left(1-U_{n}^{2}\right) \cap \operatorname{ker} P_{n+1}$; then, for any $k=1, \ldots, n$,

$$
\begin{aligned}
P_{n+1} T_{k} v & =P\left(D_{\{k\}}\right)\left(1+T_{k}\right) T_{k} v \\
& =P\left(D_{\{k\}}\right)\left(T_{k}+T_{k}^{2}\right) v \\
& =P\left(D_{\{k\}}\right)\left(1+T_{k}\right) v=P_{n+1} v=0 .
\end{aligned}
$$

Therefore $T_{k}$ maps $\operatorname{ker}\left(1-U_{n}^{2}\right) \cap \operatorname{ker} P_{n+1}$ onto itself for any $k=1, \ldots, n$. This fact implies that, for any $\sigma \in S_{n+1}$, we have $\phi(\sigma) v \in \operatorname{ker}\left(1-U_{n}^{2}\right) \cap \operatorname{ker} P_{n+1}$, and

$$
\forall k=1, \ldots, n, \forall \sigma \in S_{n+1}, \quad\left(1-T_{k}^{2}\right) \phi(\sigma) v=0 .
$$

For convenience, we fix the set $S_{n+1}$, and set $v_{i}:=\phi\left(\pi_{i}\right) v$ for $\pi_{i} \in S_{n+1}$ $\left(\pi_{1}:=\mathrm{id}\right.$ and $\left.v_{1}=v\right)$. Then the condition $P_{n+1} v=0$ takes the form

$$
\sum_{k=1}^{n !} v_{k}=0 .
$$

Finally, for any pair $i \neq j$ there exist generators $\sigma_{i_{1}}, \ldots, \sigma_{i_{m}} \in S$ such that

$$
\pi_{j}=\sigma_{i_{1}} \cdots \sigma_{i_{m}} \pi_{i}
$$

and

$$
v_{j}=T_{i_{1}} \cdots T_{i_{m}} v_{i} .
$$

Note that, if $v_{k}=T_{r} v_{l}$ for some $r=1, \ldots, n$, then $T_{r}^{2} v_{k}=v_{k}$ implies that $v_{k}-v_{l} \in \operatorname{ker}\left(1+T_{r}\right)$. Therefore, for any $i \neq j$,

$$
v_{i}-v_{j} \in \sum_{k=1}^{n} \operatorname{ker}\left(1+T_{k}\right) .
$$

In particular, for any $j=2, \ldots, n$ !,

Then from (11), we have

$$
v_{1}-v_{j} \in \sum_{k=1}^{n} \operatorname{ker}\left(1+T_{k}\right) .
$$

and therefore

$$
n ! v=n ! v_{1} \in \sum_{k=1}^{n} \operatorname{ker}\left(1+T_{k}\right),
$$

$$
v \in \sum_{k=1}^{n} \operatorname{ker}\left(1+T_{k}\right) \text {. }
$$


Remark 4. Evidently the proof does not depend on the dimension of $\mathcal{K}$. Indeed, in the case when $\mathcal{K}$ is infinite-dimensional, the linear subspace in (9) is replaced by its closure. I.e., if $\mathcal{K}$ is a separable Hilbert space and $\left\{T_{i}, i=1, \ldots, n\right\}$ are selfadjoint contractions satisfying the braid conditions, then

$$
\operatorname{ker} P_{n+1}=\overline{\sum_{k=1}^{n} \operatorname{ker}\left(1+T_{k}\right)} \text {. }
$$

As a corollary we have an improved version of the result of Bożejko and Speicher (see [2]).

Proposition 5. If the operator $T$ satisfies the braid condition, and $-1<$ $T \leq 1$, then $P_{n}>0, n \geq 2$, i.e., the Fock inner product is strictly positive, and the Fock representation acts in the whole space $\mathcal{T}(\mathcal{H})$.

Proof. Recall that if $T$ is braided and $\|T\| \leq 1$ then $P_{n} \geq 0$ (see [2]). It remains only to show that $\operatorname{ker} P_{n}=\{0\}$ for $-1<T \leq 1$. This fact trivially follows from our theorem since in this case $\operatorname{ker}(1+T)=\{0\}$.

\section{Corollaries and examples.}

We summarize the results obtained above in the following proposition.

Proposition 6. If $W(T)$ is a Wick algebra with braided operator of coefficients $T$ satisfying the norm bound $\|T\| \leq 1$, then the following three statements hold.

1. The kernel of the Fock representation is generated by the largest quadratic Wick ideal. In particular, if $-1<T \leq 1$, then the Fock representation is faithful.

2. For any $n \geq 2$ we have the inclusion $\mathcal{J}_{n} \subset \mathcal{J}_{2}$.

3. If $-1<T \leq 1$, then $W(T)$ has no non-trivial Wick ideals.

Example 1. Consider the $q$-CCR algebra based on a Hilbert space $\mathcal{H}$ and the relations

$$
\begin{aligned}
& a_{i}^{*} a_{i}=1+q a_{i} a_{i}^{*}, \quad i=1, \ldots, d, \\
& a_{i}^{*} a_{j}=q a_{j} a_{i}^{*}, \quad i \neq j, \quad 0<q<1 .
\end{aligned}
$$

We pick an orthogonal basis $\left(e_{i}\right)$ in $\mathcal{H}$, and then $T$ is determined on this basis by the formulas

$$
T e_{i} \otimes e_{j}=q e_{j} \otimes e_{i}, \quad\|T\|<1 .
$$

It is evident that $T$ is braided. Then by the proposition, we cannot have any Wick ideals in $W(T)$. 
It was proved in [2] that for braided $T$ satisfying the norm bound $\|T\|<1$, the Fock representation is bounded. Therefore we may consider the $C^{*}$ algebra generated by operators of the Fock representation.

Recall that a $*$-algebra is called $C^{*}$-representable if it can be realized as a $*$-subalgebra of a certain $C^{*}$-algebra (see for example [7]). Combining the results of Theorem 2 and Proposition 5, we obtain the following statement.

Proposition 7. If $W(T)$ is a Wick algebra with braided operator of coefficients $T$ satisfying the norm bound $\|T\|<1$, then $W(T)$ is $C^{*}$-representable.

Suppose that, in the case of braided $T$ with $\|T\|=1$ and $\operatorname{ker}(1+T) \neq$ $\{0\}$, the Fock representation is bounded. Then Theorem 2 implies that the quotient $W(T) / \mathcal{J}_{2}$ is $C^{*}$-representable.

Example 2. Consider the following type of $q_{i j}$-CCR (see [2]):

$$
\begin{aligned}
& a_{i}^{*} a_{i}=1+q_{i} a_{i} a_{i}^{*}, \quad i=1, \ldots, d, \quad 0<q_{i}<1, \\
& a_{i}^{*} a_{j}=\lambda_{i j} a_{j} a_{i}^{*}, \quad i \neq j, \quad\left|\lambda_{i j}\right|=1, \quad \lambda_{i j}=\bar{\lambda}_{i j} .
\end{aligned}
$$

The corresponding $T$ is braided, $\|T\|=1$, and

$$
\operatorname{ker}(1+T)=\left\langle a_{j} a_{i}-\lambda_{i j} a_{i} a_{j}, i<j\right\rangle .
$$

Moreover, the Fock representation of this algebra is bounded. Then, as noted above, the $*$-algebra generated by the relations

$$
\begin{aligned}
a_{i}^{*} a_{i} & =1+q_{i} a_{i} a_{i}^{*}, \quad i=1, \ldots, d, \quad 0<q_{i}<1, \\
a_{i}^{*} a_{j} & =\lambda_{i j} a_{j} a_{i}^{*}, \quad i \neq j, \quad\left|\lambda_{i j}\right|=1, \quad \lambda_{i j}=\bar{\lambda}_{i j}, \\
a_{j} a_{i} & =\lambda_{i j} a_{i} a_{j}, \quad i<j
\end{aligned}
$$

is $C^{*}$-representable.

A description of the irreducible representations of these relations can be found for example in [8, Sec. 2.4].

Note that, if $\|T\|=1$, then the operators of the Fock representation can be unbounded.

Example 3. Consider the following Wick algebra:

$$
\begin{aligned}
& a_{i}^{*} a_{i}=1+a_{i} a_{i}^{*}, \quad i=1, \ldots, d, \\
& a_{i}^{*} a_{j}=q a_{j} a_{i}^{*}, \quad i \neq j, \quad-1<q<1 .
\end{aligned}
$$

The corresponding $T$ is determined by the formulas

$$
T e_{i} \otimes e_{i}=e_{i} \otimes e_{i}, \quad T e_{j} \otimes e_{i}=q e_{i} \otimes e_{j}, i \neq j, i=1, \ldots, d .
$$

It is easy to see that $T$ is braided and $-1<T \leq 1$. So, the Fock representation of this algebra is faithful. Note that, if we consider the complement of $\mathcal{T}(\mathcal{H})$ with respect to the Fock inner product, then the operators of the Fock representation are unbounded.

For the definition and properties of representations of $*$-algebras by unbounded operators, see for example $[\mathbf{1 1}]$. 
Unbounded representations of Wick algebras will be considered in more detail later.

Acknowledgements. Yu. Samoulenko and Dan. Proskurin express their gratitude to Prof. Vasyl L. Ostrovskyı̆ and Stanislav Popovych for their attention and helpful discussions. We also thank Brian Treadway for proofreading and polishing.

\section{References}

[1] N. Bourbaki, Eléments de mathematique, Fasc. XXXIV: Groupes et algebres de Lie, Chap. 4, 5, 6, Hermann, Paris, 1968.

[2] M. Bożejko and R. Speicher, Completely positive maps on Coxeter groups, deformed commutation relations, and operator spaces, Math. Ann., 300 (1994), 97-120.

[3] K. Dykema and A. Nica, On the Fock representation of the q-commutation relations, J. Reine Angew. Math., 440 (1993), 201-212.

[4] O.W. Greenberg, Particles with small violations of Fermi or Bose statistics, Phys. Rev. D (3), 43 (1991), 4111-4120.

[5] P.E.T Jørgensen, L.M. Schmitt and R.F. Werner, q-canonical commutation relations and stability of the Cuntz algebra, Pacific J. Math., 165(1) (1994), 131-151.

[6] P.E.T. Jørgensen, L.M. Schmitt, and R.F. Werner, Positive representations of general commutation relations allowing Wick ordering, J. Funct. Anal., 134 (1995), 33-99.

[7] E.C. Lance, Finitely-presented $C^{*}$-algebras, Operator Algebras and Applications, NATO Adv. Sci. Inst. Ser. C Math. Phys. Sci., Vol. 495, Kluwer Acad. Publ., Dordrecht, 1997, 255-266.

[8] V. Ostrovskyı̆ and Yu. Samoĭlenko, Introduction to the Theory of Representations of Finitely Presented *-Algebras, I: Representations by Bounded Operators, The Gordon and Breach Publishing Group, London, 1999.

[9] D. Proskurin, Homogeneous ideals in Wick *-algebras, Proc. Amer. Math. Soc., 126(11) (1998), 3371-3376.

[10] W. Pusz and S.L. Woronowicz, Twisted second quantization, Rep. Math. Phys., 27 (1989), 231-257.

[11] K. Schmüdgen, Unbounded Operator Algebras and Representation Theory, Operator Theory: Advances and Applications, Vol. 37, Birkhäuser, Basel, 1990.

Received May 25, 1999. The first author was partially supported by NSF grant no. DMS9700130. The third author was partially supported by CRDF grant no. UM1-311

Department of Mathematics

The UNIVERSity OF IOWA

IowA City, IowA 52242-1419

E-mail address: jorgen@math.uiowa.edu

INSTITUTE OF MATHEMATICS

Ukrainian National ACADEmy of Sciences

TERESHCHINKIVS'KA, 3

KIEV, 252601

UKRAINE 
E-mail address: prosk@imath.kiev.ua

Institute OF MATHEMATiCs

Ukrainian National ACADEMy of Sciences

TereshCHINKIVS'KA, 3

KIEV, 252601

UKRAINE

E-mail address: Yurii_Sam@imath.kiev.ua 